\title{
Effect of Growth Regulators and Growth Media on the Rhizogenesis of Some Genotypes of Rose through Stem Cuttings
}

\author{
Sonam Dawa ${ }^{1 *}$, Z.A. Rather ${ }^{1}$, Tashi Stobgais ${ }^{2}$, Tsering Angdus ${ }^{2}$, \\ Stanzin Lakdan ${ }^{3}$ and Phunstog Tundup ${ }^{3}$ \\ ${ }^{1}$ Division of FLA, India \\ ${ }^{2}$ National Research Institute for Sowa-Rigpa-Leh, Division of Soil Science, \\ Dr. P.D.K.V.-Akola, Maharashtra, India \\ ${ }^{3}$ Division of Soil Science, SKUAST-K, Shalimar-190025, Kolkata, India \\ *Corresponding author
}

\section{Keywords}

Growing medium, IBA, NAA,

Rootstocks, Rooting, Rose

\section{Article Info}

Accepted:

10 December 2017

Available Online:

10 January 2018

\section{A B S T R A C T}

This study was initiated to observe the effect of growth regulators (IBA 500, 1000, 1500, NAA $500,1000,1500 \mathrm{ppm}$ ) and growing medium combinations (Sand, sand $85 \%+$ manure $15 \%$, sand $70 \%+$ manure $30 \%$, sand $70 \%+$ manure $15 \%+$ soil $15 \%$, sand $85 \%+$ soil $15 \%$ ) on the rooting of three rootstocks of rose (Rosa indica, Rosa banksiae and Rosa bourboniana). Main effect of growth regulators and genotype was significant on majority of rooting characteristics. IBA $(1000 \mathrm{ppm})$ recorded minimum days to root initiation (23.33), maximum rooting $(72.22 \%)$, root length $(6.42 \mathrm{~cm})$ and field survival $(82.38 \%)$. Among the genotype, Rosa indica performed best recording maximum rooting (75.24\%), primary root number $(9.48)$, root length $(6.40 \mathrm{~cm})$, new leaf growth on cuttings $(59.05 \%)$ and field survival (96.98\%) followed by Rosa banksiae. Performance of Rosa bourboniana was poor recording lowest values for all these parameters. Interaction effects were significant. NAA produced superior results in Rosa indica while, IBA gave promising results in Rosa banksiae and Rosa bourboniana. Influence of growing medium significantly improved various rooting characteristics of all the three genotypes. Superior results were obtained with $\mathrm{M}_{3}$ medium (sand $70 \%+$ manure 30\%) which, recorded minimum days to root initiation (22.13), maximum rooting (83.33\%), primary root number (15.52), root length $(8.88 \mathrm{~cm})$, new leaf growth $(51.11 \%)$ and field survival $(85.46 \%)$. Among the genotypes, Rosa indica consistently gave better results recording minimum days to root initiation (22.70), maximum rooting (90.00\%), primary root number (21.21), root length $(10.10 \mathrm{~cm})$, new leaf growth $(47.33 \%)$ and field survival $(97.78 \%)$.

\section{Introduction}

Rose is the best known and most popular of all the garden flowers throughout the world. Among the top three cut flowers in the international imports, rose occupies the second position. The Netherland is the leading exporter of rose followed by Columbia and Israel (Bose et al., 2002). Now in India, rose is being considered the principal cut flower crop and is grown in an approximately 20 per cent of the total area under ornamental plants 
(Singh and Dadlani, 1993). Roses are conveniently propagated by cuttings, budding, grafting and layering. Among these, the use of stem cuttings is most easy and common method of growing roses (Anderson and Woods, 1999). Propagation through cuttings is the simplest way to increase the desirable rose cultivars but success rate is limited in many types due to failure in root formation. Plant growth regulators could promote rooting in many ornamental plants including roses (Pandey and Sinha, 1997). Synthetic auxins like IBA and NAA are the principle auxins which have been used for rooting of cuttings and majority of plant species are responsive to them (Arteca, 1996).

The performance of varieties depends to a large extent on the availability of rootstock suitable for local soil and climatic conditions. Kaicker and Dhayani (1986) evaluated twelve indigenous and exotic rootstocks of rose and found Rosa indica var. odorata suitable for North Indian plains particularly for saline soils and Rosa bourboniana suitable for whole country.

Commercial cultivation of rose in Jammu \& Kashmir state depends upon the availability of planting material of good cut flower cultivars and their suitability to the local environment. Performance of the new cultivars can be increased to some extent if budded upon the locally available rootstocks. Thus multiplication of local rootstocks is very important for commercialization of rose in the state so that quality planting material budded on suitable rootstocks can be distributed among the growers for large scale cultivation.

\section{Materials and Methods}

The present investigations were carried out at the Research Farm of Division of Floriculture, Medicinal and Aromatic Plants, SKUAST(K) Shalimar Campus, Srinagar during the year 2010. The studies were conducted to standardize the growth regulator concentration and rooting medium formulation for improving the rooting characteristics of three rootstocks of rose (Rosa indica, Rosa bourboniana and Rosa banksiae var. lutea). Sand is generally used as a medium for rooting of large number of cuttings.

Two different experiments were designed to study the rooting behavior of different genotypes of rose under the influence of growth regulators (IBA and NAA) and growing media combinations. Mature plants of three genotypes of rose growing at the experimental farm under open conditions for the last 5 years were selected as stock plants for collecting semi hardwood cutting material for the experiments.

The experiments were laid out in the underground cellars (propagation chambers) $50 \mathrm{~cm}$ deep. The cellars were covered with transparent polythene from top using wooden frames for providing support to the sheets. Tin trays were filled with rooting medium (washed river sand) and the medium was drenched with $0.02 \%$ bavistin five days before inserting the cuttings into the medium. Sand filled trays were put in the underground cellars for planting of cuttings.

The semi-hard wood cuttings of different rootstocks (Rosa indica, Rosa bourboniana and Rosa banksiae var. lutea) of rose consisting of 4 nodes with leaves intact on each node were taken from the middle portion of the vigorously growing shoots during morning hours. Each cutting was prepared by giving a flat round cut at the basal portion just below the lower bud and another slanting cut was made at the top $2.0-3.0 \mathrm{~cm}$ away from the top bud. Lower leaves on the cuttings were removed to avoid their contact with the rooting medium. Cuttings were given a quick dip for 10 seconds in freshly prepared auxin 
solution (500, 1000 and $1500 \mathrm{ppm})$. Cuttings under control were dipped in distilled water for same duration.

Treated cuttings were immediately planted in the sand filled trays already set in cellars with the help of a dibbler at a distance of $5.0 \times 10$ $\mathrm{cm}$ and $1.5 \mathrm{~cm}$ depth. The rooting media used was moistened beforehand so that only a light overhead watering was needed to settle the cuttings without risk of leaching off of the rooting chemical. Similarly treated cuttings were planted vertically in growing medium filled plastic glasses with the help of a dibbler and slightly watered. After planting of cuttings in the medium, the glasses were kept in the underground cellars covered with polythene sheets and watered as and when necessary.

Second experiments were conducted to find out the effect of combination of sand with other media (garden soil and farmyard manure) on the various rooting parameters of rose. Different combinations of growing media (sand, garden soil, well rotten farmyard manure) were made on volume basis. One liter plastic bucket was used to mix different quantities of growing medium. Required quantity of each medium for a particular treatment combination was measured with the help of plastic bucket and put on the plastic sheet. Different medium components were then thoroughly mixed and filled in the plastic glasses upto brim. Few holes were made at the bottom of each glass to ensure free drainage. Medium filled plastic glasses were thoroughly watered with the $0.02 \%$ bavistin solution and kept under semi-shade for a few days. Semi hardwood cuttings treated with IBA (1000 ppm) were planted in these glasses and kept under a poly tunnel.

The data recorded on different rooting parameters was subjected to analysis of variance for completely randomized design with three replications (Gomez and Gomez,
1983) using Minitab statistical package. To satisfy model assumptions for analysis of variance, percentage data was subjected to angular transformation as suggested by Steel and Torrie (1981). Mean comparison was performed using Least Significant Difference (LSD) test.

\section{Results and Discussion}

Success of rooting in cuttings depends upon the species and cultivar, condition of the cutting wood, type of cuttings, season and many other factors (Hartmann et al., 2002). Synthetic root promoting chemicals that have been found most reliable in stimulating adventitious root production in cuttings (Arteca, 1996). Data collected from the first experiment revealed that growth regulators and genotype had a significant effect on various rooting characteristics of rose. Minimum days to root initiation (23.33 days) were recorded with IBA (1000 ppm) in comparison to control, which took maximum time (26.00 days). The decrease in time taken to root initiation may be attributed to the fact that application of exogenous growth regulators might have supplemented endogenous auxin levels and brought about certain anatomical and physiological changes in the cuttings leading to early root initiation. Among the genotypes, minimum time (22.81days) was taken by Rosa indica and maximum (25.71 days) by Rosa bourboniana (Table 1). Early root initiation in some genotypes might be due to presence of high carbohydrates in association with high phenolic compounds in them. These compounds get reduced at the time of root initiation and favour rooting in cuttings (Patil and Shirol, 1991). Maximum rooting $(72.22 \%)$ was obtained in cuttings treated with IBA 1000 ppm, while minimum rooting was recorded in control $(55.56 \%)$. IBA was found more effective than NAA in improving rooting percentage of cuttings. Yashaswini et al., 
(2011) also obtained maximum rooting percentage with $1000 \mathrm{ppm}$ IBA application in Premna integrifolia. The increase in percentage of rooting as a result of IBA treatments may be due to the fact that IBA helps in mobilization of reserve food materials, elongation of meristematic cells and differentiation of cambial initial into root primordial (Nanda, 1975). Increase in the number of root primordia and hence increased rooting percentage has been reported by Rolston et al., (1996) following treatment of cuttings with IBA in Mussaendas cv. Rosea. Among the various genotypes, highest rooting (75.24\%) was recorded with Rosa indica and was significantly superior to Rosa banksiae and Rosa bourboniana. Differences in the rooting capacity of genotypes under study might be attributed to genetic composition of the genotypes. Significant interaction yielded 93.33\% rooting in Rosa indica with NAA (1000 ppm).

There was a significant increase in the number of primary roots with the application of growth regulators (Table 2). Highest number of primary roots (8.59) was recorded in cuttings treated with NAA $1000 \mathrm{ppm}$ and lowest number of roots (3.88) was recorded in control cuttings, which received no growth regulator treatment. Superiority of NAA over IBA was due to more number of roots produced by Rosa indica, which increased the overall mean; otherwise IBA 1000 was found better for improving the primary root number in other two genotypes. Exogenous auxin treatment have been reported to increase the number of root primordia in the basal part of the cuttings, which led to increased rooting and root number (Rolston et al., 1996).

Among the genotypes, Rosa indica recorded maximum number (9.48) of primary roots as compared to Rosa banksiae (6.92), while minimum number of roots (3.03) was recorded under control in Rosa bourboniana.
Maximum length of primary roots $(6.42 \mathrm{~cm})$ was recorded with IBA $1000 \mathrm{ppm}$ and minimum of $4.26 \mathrm{~cm}$ was observed under control. Among the genotypes, Rosa indica recorded maximum root length of $6.40 \mathrm{~cm}$ and minimum of $3.29 \mathrm{~cm}$ in Rosa bourboniana. Significant interaction yielded maximum root length of $7.67 \mathrm{~cm}$ in Rosa indica with the application of 1000 ppm NAA (Table 2). Exogenous application of auxin may have caused hydrolysis and translocation of carbohydrate and nitrogenous substances present at the base of cuttings, which resulted in increased length of roots (Singh et al., 2003).

Maximum number of cuttings with new leaves $(43.33 \%)$ was recorded in NAA 1000 ppm, which was at par with IBA 1000 ppm $(42.22 \%)$. Minimum value for this parameter $(30.00 \%)$ was recorded in cuttings under control cuttings (Table 3). Development of new leaves in cuttings might be associated with the increased root number and length in treated cuttings, which helped them in better nutrient and moisture utilization from the growing medium and hence more growth in the form of new leaves.

Among the various genotypes, Rosa indica recorded maximum number of cuttings with new leaves $(59.05 \%)$ and minimum $(20.95 \%)$ was observed in Rosa bourboniana. Superiority of Rosa indica with respect to development of new leaves might be attributed to better utilization of nutrients from the growing medium by dent of its maximum root number and root length. Significant interaction resulted in the production of maximum cuttings with new leaves $(80.00 \%)$ in Rosa indica when treated with NAA 1000 ppm.

Survival of the rooted cuttings under field conditions is a major objective, which determines the overall success of any plant propagation project. 
Table.1 Effect of growth regulators on the days taken to root initiation and rooting in some genotypes of rose

\begin{tabular}{|c|c|c|c|c|c|c|c|c|c|}
\hline \multirow{3}{*}{$\begin{array}{l}\text { Growth } \\
\text { Regulator }\end{array}$} & \multirow{3}{*}{$\begin{array}{l}\text { Concentration } \\
\text { (ppm) }\end{array}$} & \multicolumn{8}{|c|}{ Genotype } \\
\hline & & \multicolumn{4}{|c|}{ Days taken to root initiation } & \multicolumn{4}{|c|}{ Rooting (\%) } \\
\hline & & $\begin{array}{c}\text { Rosa } \\
\text { bourboniana }\end{array}$ & $\begin{array}{c}\text { Rosa } \\
\text { banksiae }\end{array}$ & $\begin{array}{l}\text { Rosa } \\
\text { indica }\end{array}$ & Mean & $\begin{array}{c}\text { Rosa } \\
\text { bourboniana }\end{array}$ & $\begin{array}{c}\text { Rosa } \\
\text { banksiae }\end{array}$ & $\begin{array}{l}\text { Rosa } \\
\text { indica }\end{array}$ & Mean \\
\hline Control & 000 & 28.00 & 26.00 & 24.00 & 26.00 & $\begin{array}{c}33.33 \\
(35.22)\end{array}$ & $\begin{array}{c}63.33 \\
(52.78)\end{array}$ & $\begin{array}{l}70.00 \\
(56.9)\end{array}$ & $\begin{array}{l}55.56 \\
(48.3)\end{array}$ \\
\hline IBA & 500 & 25.00 & 24.00 & 22.00 & 23.67 & $\begin{array}{c}56.67 \\
(48.85)\end{array}$ & $\begin{array}{c}73.33 \\
(59.01)\end{array}$ & $\begin{array}{l}80.00 \\
(63.9)\end{array}$ & $\begin{array}{l}70.00 \\
(57.2)\end{array}$ \\
\hline IBA & 1000 & 24.00 & 23.00 & 23.00 & 23.33 & $\begin{array}{c}63.33 \\
(52.78)\end{array}$ & $\begin{array}{c}83.33 \\
(66.15)\end{array}$ & $\begin{array}{l}70.00 \\
(56.9)\end{array}$ & $\begin{array}{l}72.22 \\
(58.6)\end{array}$ \\
\hline IBA & 1500 & 24.33 & 25.00 & 24.00 & 24.44 & $\begin{array}{c}50.00 \\
(45.00)\end{array}$ & $\begin{array}{c}66.67 \\
(54.78)\end{array}$ & $\begin{array}{l}56.67 \\
(48.8)\end{array}$ & $\begin{array}{l}57.79 \\
(49.5)\end{array}$ \\
\hline NAA & 500 & 27.00 & 25.00 & 21.00 & 24.33 & $\begin{array}{c}50.00 \\
(45.00)\end{array}$ & $\begin{array}{c}56.67 \\
(48.85)\end{array}$ & $\begin{array}{l}83.33 \\
(66.1)\end{array}$ & $\begin{array}{l}63.33 \\
(53.3)\end{array}$ \\
\hline NAA & 1000 & 25.00 & 24.00 & 23.00 & 24.00 & $\begin{array}{c}43.33 \\
(41.16)\end{array}$ & $\begin{array}{c}70.00 \\
(56.99)\end{array}$ & $\begin{array}{l}93.33 \\
(77.7)\end{array}$ & $\begin{array}{l}68.88 \\
(58.6)\end{array}$ \\
\hline NAA & 1500 & 26.67 & 24.00 & 22.67 & 24.44 & $\begin{array}{c}43.33 \\
(41.16)\end{array}$ & $\begin{array}{c}50.00 \\
(45.00)\end{array}$ & $\begin{array}{l}73.33 \\
(59.7)\end{array}$ & $\begin{array}{l}55.57 \\
(48.6)\end{array}$ \\
\hline Mean & & 25.71 & 24.43 & 22.81 & & $\begin{array}{c}48.57 \\
(44.16)\end{array}$ & $\begin{array}{c}66.19 \\
(54.79)\end{array}$ & $\begin{array}{c}75.24 \\
(61.48)\end{array}$ & \\
\hline C.D. $(\mathrm{p} \leq 0 . \mathrm{C}$ & $\begin{array}{l}\text { Growtl } \\
\text { Genoty } \\
\text { Interac }\end{array}$ & $\begin{array}{l}\text { regulator }=1.4 \\
\mathrm{e}=0.922 \\
\mathrm{on}=\mathrm{NS}\end{array}$ & & & & & & & $\begin{array}{l}3.081 \\
3.326 \\
3.799\end{array}$ \\
\hline
\end{tabular}

Table.2 Effect of growth regulators on the primary root number and root length in some genotypes of rose

\begin{tabular}{|c|c|c|c|c|c|c|c|c|c|}
\hline \multirow{3}{*}{$\begin{array}{l}\text { Growth } \\
\text { Regulator }\end{array}$} & \multirow{3}{*}{$\begin{array}{l}\text { Concentration } \\
(\mathrm{ppm})\end{array}$} & \multicolumn{8}{|c|}{ Genotype } \\
\hline & & \multicolumn{4}{|c|}{ Primary root number } & \multicolumn{4}{|c|}{ Root length (cm) } \\
\hline & & $\begin{array}{c}\text { Rosa } \\
\text { bourboniana }\end{array}$ & $\begin{array}{c}\text { Rosa } \\
\text { banksiae }\end{array}$ & $\begin{array}{l}\text { Rosa } \\
\text { indica }\end{array}$ & Mean & $\begin{array}{c}\text { Rosa } \\
\text { bourboniana }\end{array}$ & $\begin{array}{c}\text { Rosa } \\
\text { banksiae }\end{array}$ & $\begin{array}{l}\text { Rosa } \\
\text { indica }\end{array}$ & Mean \\
\hline Control & 000 & 2.23 & 4.60 & 4.80 & 3.88 & 3.03 & 4.20 & 5.53 & 4.26 \\
\hline IBA & 500 & 3.20 & 8.20 & 6.67 & 6.02 & 3.27 & 6.20 & 6.10 & 5.19 \\
\hline IBA & 1000 & 4.47 & 9.13 & 7.33 & 6.98 & 3.87 & 8.60 & 6.80 & 6.42 \\
\hline IBA & 1500 & 3.03 & 7.30 & 6.00 & 5.44 & 3.77 & 7.80 & 5.20 & 5.59 \\
\hline NAA & 500 & 2.40 & 7.60 & 10.80 & 6.93 & 3.10 & 5.00 & 7.10 & 5.07 \\
\hline NAA & 1000 & 3.03 & 6.20 & 16.53 & 8.59 & 3.03 & 5.60 & 7.67 & 5.43 \\
\hline NAA & 1500 & 2.83 & 5.40 & 14.20 & 7.48 & 2.97 & 4.90 & 6.40 & 4.76 \\
\hline \multicolumn{2}{|l|}{ Mean } & 3.03 & 6.92 & 9.48 & & 3.29 & 6.04 & 6.40 & \\
\hline \multicolumn{2}{|c|}{ C.D. $(p \leq 0.05)$} & $\begin{array}{l}\text { regulator } \\
\text { pe = } \\
\text { ion = }\end{array}$ & & & & & & $\begin{array}{l}0.56 \\
0.37 \\
0.98\end{array}$ & \\
\hline
\end{tabular}


Table.3 Effect of growth regulators on the cuttings with new leaves and field survival in some genotypes of rose

\begin{tabular}{|c|c|c|c|c|c|c|c|c|c|}
\hline \multirow{3}{*}{$\begin{array}{l}\text { Growth } \\
\text { Regulator }\end{array}$} & \multirow{3}{*}{$\begin{array}{l}\text { Concentration } \\
\text { (ppm) }\end{array}$} & \multicolumn{8}{|c|}{ Genotype } \\
\hline & & \multicolumn{4}{|c|}{ Cuttings with new leaves (\%) } & \multicolumn{4}{|c|}{ Field survival (\%) } \\
\hline & & $\begin{array}{c}\text { Rosa } \\
\text { bourboniana }\end{array}$ & $\begin{array}{c}\text { Rosa } \\
\text { banksiae }\end{array}$ & $\begin{array}{l}\text { Rosa } \\
\text { indica }\end{array}$ & Mean & $\begin{array}{c}\text { Rosa } \\
\text { bourboniana }\end{array}$ & $\begin{array}{c}\text { Rosa } \\
\text { banksiae }\end{array}$ & $\begin{array}{l}\text { Rosa } \\
\text { indica }\end{array}$ & Mean \\
\hline Control & 000 & $\begin{array}{c}13.33 \\
(21.15)\end{array}$ & $\begin{array}{c}30.00 \\
(33.00)\end{array}$ & $\begin{array}{c}46.67 \\
(43.08)\end{array}$ & $\begin{array}{c}30.00 \\
(32.41)\end{array}$ & $\begin{array}{c}30.16 \\
(33.29)\end{array}$ & $\begin{array}{c}79.26 \\
(62.91)\end{array}$ & $\begin{array}{l}89.63 \\
(71.22)\end{array}$ & $\begin{array}{c}66.35 \\
(55.81)\end{array}$ \\
\hline IBA & 500 & $\begin{array}{l}20.00 \\
(26.07)\end{array}$ & $\begin{array}{c}30.00 \\
(33.00)\end{array}$ & $\begin{array}{c}56.67 \\
(48.85)\end{array}$ & $\begin{array}{l}35.56 \\
(35.97)\end{array}$ & $\begin{array}{c}47.50 \\
(43.56)\end{array}$ & $\begin{array}{c}92.96 \\
(77.36)\end{array}$ & $\begin{array}{l}96.29 \\
(83.51)\end{array}$ & $\begin{array}{c}78.92 \\
(68.14)\end{array}$ \\
\hline IBA & 1000 & $\begin{array}{c}33.33 \\
(35.22)\end{array}$ & $\begin{array}{c}40.00 \\
(39.23)\end{array}$ & $\begin{array}{c}53.33 \\
(47.01)\end{array}$ & $\begin{array}{c}42.22 \\
(40.49)\end{array}$ & $\begin{array}{c}54.17 \\
(47.41)\end{array}$ & $\begin{array}{c}96.29 \\
(83.51)\end{array}$ & $\begin{array}{c}96.67 \\
(83.85)\end{array}$ & $\begin{array}{l}82.38 \\
(71.59)\end{array}$ \\
\hline IBA & 1500 & $\begin{array}{c}26.67 \\
(30.99)\end{array}$ & $\begin{array}{c}26.67 \\
(30.99)\end{array}$ & $\begin{array}{c}46.67 \\
(43.01)\end{array}$ & $\begin{array}{c}33.33 \\
(35.02)\end{array}$ & $\begin{array}{c}47.50 \\
(43.56)\end{array}$ & $\begin{array}{c}89.26 \\
(74.48)\end{array}$ & $\begin{array}{l}96.29 \\
(83.51)\end{array}$ & $\begin{array}{c}77.68 \\
(67.18)\end{array}$ \\
\hline NAA & 500 & $\begin{array}{c}16.67 \\
(23.85)\end{array}$ & $\begin{array}{c}26.67 \\
(30.99)\end{array}$ & $\begin{array}{c}66.67 \\
(54.99)\end{array}$ & $\begin{array}{c}36.67 \\
(36.67)\end{array}$ & $\begin{array}{c}40.54 \\
(39.42)\end{array}$ & $\begin{array}{c}86.67 \\
(72.29)\end{array}$ & $\begin{array}{l}100.00 \\
(90.00)\end{array}$ & $\begin{array}{c}75.77 \\
(67.24)\end{array}$ \\
\hline NAA & 1000 & $\begin{array}{l}20.00 \\
(26.07)\end{array}$ & $\begin{array}{c}30.00 \\
(33.00)\end{array}$ & $\begin{array}{c}80.00 \\
(63.93)\end{array}$ & $\begin{array}{c}43.33 \\
(41.00)\end{array}$ & $\begin{array}{c}43.33 \\
(41.15)\end{array}$ & $\begin{array}{c}90.47 \\
(79.23)\end{array}$ & $\begin{array}{l}100.00 \\
(90.00)\end{array}$ & $\begin{array}{c}77.94 \\
(70.13)\end{array}$ \\
\hline NAA & 1500 & $\begin{array}{l}16.67 \\
(23.85)\end{array}$ & $\begin{array}{c}26.68 \\
(30.99)\end{array}$ & $\begin{array}{c}63.33 \\
(52.78)\end{array}$ & $\begin{array}{c}35.56 \\
(35.88)\end{array}$ & $\begin{array}{c}40.47 \\
(39.40)\end{array}$ & $\begin{array}{c}86.29 \\
(68.51)\end{array}$ & $\begin{array}{l}100.00 \\
(90.00)\end{array}$ & $\begin{array}{c}75.59 \\
(65.99)\end{array}$ \\
\hline \multicolumn{2}{|l|}{ Mean } & $\begin{array}{l}20.95 \\
(26.74)\end{array}$ & $\begin{array}{c}30.00 \\
(33.03)\end{array}$ & $\begin{array}{l}59.05 \\
(50.53)\end{array}$ & & $\begin{array}{c}43.38 \\
(41.12)\end{array}$ & $\begin{array}{c}88.74 \\
(74.04)\end{array}$ & $\begin{array}{l}96.98 \\
(84.58)\end{array}$ & \\
\hline \multicolumn{2}{|c|}{ C.D. $(p \leq 0.05)$} & $\begin{array}{l}\text { rowth regulator } \\
\text { Genotype = } \\
\text { Interaction }\end{array}$ & $=$ & \multicolumn{2}{|c|}{$\begin{array}{l}5.089 \\
3.332\end{array}$} & & & & $\begin{array}{c}8.321 \\
5.448 \\
\text { NS }\end{array}$ \\
\hline
\end{tabular}

Table.4 Effect of growing media on the days taken to root initiation and rooting (\%) in some genotypes of rose

\begin{tabular}{|c|c|c|c|c|c|c|c|c|}
\hline \multirow[t]{3}{*}{ Growing medium } & \multicolumn{8}{|c|}{ Genotype } \\
\hline & \multicolumn{4}{|c|}{ Days taken to root initiation } & \multicolumn{4}{|c|}{ Rooting (\%) } \\
\hline & $\begin{array}{c}\text { Rosa } \\
\text { bourboniana }\end{array}$ & $\begin{array}{c}\text { Rosa } \\
\text { banksiae }\end{array}$ & $\begin{array}{l}\text { Rosa } \\
\text { indica }\end{array}$ & Mean & $\begin{array}{c}\text { Rosa } \\
\text { bourboniana }\end{array}$ & $\begin{array}{c}\text { Rosa } \\
\text { banksiae }\end{array}$ & $\begin{array}{l}\text { Rosa } \\
\text { indica }\end{array}$ & Mean \\
\hline M1= Sand $(100 \%)$ & 25.20 & 24.60 & 22.00 & 23.93 & $\begin{array}{c}50.00 \\
(45.00)\end{array}$ & $\begin{array}{l}70.00 \\
(56.99)\end{array}$ & $\begin{array}{l}86.67 \\
(72.29)\end{array}$ & $\begin{array}{r}68.89 \\
(58.09)\end{array}$ \\
\hline $\begin{array}{l}\text { M2=Sand+Manure } \\
(85 \%+15 \%)\end{array}$ & 24.10 & 24.80 & 21.20 & 23.37 & $\begin{array}{c}63.33 \\
(52.78)\end{array}$ & $\begin{array}{c}80.00 \\
(63.93)\end{array}$ & $\begin{array}{l}96.67 \\
(83.86)\end{array}$ & $\begin{array}{r}80.00 \\
(66.85)\end{array}$ \\
\hline $\begin{array}{l}\text { M3=Sand+Manure } \\
(70 \%+30 \%)\end{array}$ & 23.40 & 23.00 & 20.00 & 22.13 & $\begin{array}{l}70.00 \\
(56.99)\end{array}$ & $\begin{array}{c}83.33 \\
(66.15)\end{array}$ & $\begin{array}{c}96.67 \\
(83.86)\end{array}$ & $\begin{array}{r}83.33 \\
(68.99)\end{array}$ \\
\hline $\begin{array}{l}\text { M4=Sand+Manure+Soil } \\
(70 \%+15 \%+15 \%)\end{array}$ & 24.40 & 25.80 & 24.10 & 24.77 & $\begin{array}{c}56.67 \\
(48.85)\end{array}$ & $\begin{array}{c}63.33 \\
(52.86)\end{array}$ & $\begin{array}{l}90.00 \\
(75.00)\end{array}$ & $\begin{array}{r}70.00 \\
(58.90)\end{array}$ \\
\hline $\begin{array}{l}\text { M5 = Sand+Soil } \\
(85 \%+15 \%)\end{array}$ & 28.00 & 26.80 & 26.20 & 27.00 & $\begin{array}{c}36.67 \\
(27.23)\end{array}$ & $\begin{array}{c}46.67 \\
(43.08)\end{array}$ & $\begin{array}{c}80.00 \\
(63.93)\end{array}$ & $\begin{array}{c}54.44 \\
(48.08)\end{array}$ \\
\hline Mean & 25.02 & 25.00 & 22.70 & & $\begin{array}{c}55.33 \\
(48.17)\end{array}$ & $\begin{array}{c}68.67 \\
(56.60)\end{array}$ & $\begin{array}{c}90.00 \\
(75.79)\end{array}$ & \\
\hline C.D. $(p \leq 0.05)$ & $\begin{array}{l}\text { Growing mediu } \\
\text { Genotype } \\
\text { Interaction }\end{array}$ & $\begin{array}{l}= \\
= \\
=\end{array}$ & & $\begin{array}{l}212 \\
.939 \\
\text { NS }\end{array}$ & & & & $\begin{array}{l}932 \\
144 \\
\text { NS }\end{array}$ \\
\hline
\end{tabular}


Table.5 Effect of growing media on the primary root number and root length in some Genotypes of rose

\begin{tabular}{|c|c|c|c|c|c|c|c|c|}
\hline \multirow[t]{3}{*}{ Growing medium } & \multicolumn{8}{|c|}{ Genotype } \\
\hline & \multicolumn{4}{|c|}{ Primary root number } & \multicolumn{4}{|c|}{ Root length $(\mathrm{cm})$} \\
\hline & $\begin{array}{c}\text { Rosa } \\
\text { bourboniana }\end{array}$ & $\begin{array}{c}\text { Rosa } \\
\text { banksiae }\end{array}$ & $\begin{array}{l}\text { Rosa } \\
\text { indica }\end{array}$ & Mean & $\begin{array}{c}\text { Rosa } \\
\text { bourboniana }\end{array}$ & $\begin{array}{c}\text { Rosa } \\
\text { banksiae }\end{array}$ & $\begin{array}{l}\text { Rosa } \\
\text { indica }\end{array}$ & Mean \\
\hline M1= Sand (100 \%) & 6.33 & 4.23 & 18.43 & 9.67 & 4.60 & 5.87 & 9.57 & 6.68 \\
\hline $\begin{array}{l}\text { M2=Sand+Manure } \\
(85 \%+15 \%)\end{array}$ & 11.93 & 6.77 & 24.07 & 14.26 & 7.13 & 6.87 & 11.10 & 8.37 \\
\hline $\begin{array}{l}\text { M3=Sand+Manure } \\
(70 \%+30 \%)\end{array}$ & 12.57 & 8.47 & 25.53 & 15.52 & 8.23 & 7.13 & 11.27 & 8.88 \\
\hline $\begin{array}{l}\text { M4=Sand+Manure+Soil } \\
(70 \%+15 \%+15 \%)\end{array}$ & 5.23 & 6.37 & 21.37 & 10.99 & 4.13 & 6.07 & 10.03 & 6.74 \\
\hline $\begin{array}{l}\text { M5 = Sand+Soil } \\
(85 \%+15 \%)\end{array}$ & 4.13 & 3.63 & 16.63 & 8.13 & 3.37 & 4.13 & 8.53 & 5.34 \\
\hline Mean & 8.04 & 5.89 & 21.21 & & 5.49 & 6.01 & 10.10 & \\
\hline C.D. $(p \leq 0.05)$ & \multicolumn{2}{|l|}{$\begin{array}{l}\text { Genotype } \\
\text { Interaction }\end{array}$} & $\begin{array}{l}= \\
= \\
=\end{array}$ & $\begin{array}{l}0.909 \\
0.703 \\
1.573\end{array}$ & & & & $\begin{array}{c}0.524 \\
0.406 \\
0.907\end{array}$ \\
\hline
\end{tabular}

Table.6 Effect of growing medium on the cuttings with new leaves and field survival in some genotypes of rose

\begin{tabular}{|c|c|c|c|c|c|c|c|c|}
\hline \multirow[t]{3}{*}{ Growing medium } & \multicolumn{8}{|c|}{ Genotype } \\
\hline & \multicolumn{4}{|c|}{ Cuttings with new leaves (\%) } & \multicolumn{4}{|c|}{ Field survival (\%) } \\
\hline & $\begin{array}{c}\text { Rosa } \\
\text { bourboniana }\end{array}$ & $\begin{array}{c}\text { Rosa } \\
\text { banksiae }\end{array}$ & $\begin{array}{l}\text { Rosa } \\
\text { indica }\end{array}$ & Mean & $\begin{array}{c}\text { Rosa } \\
\text { bourboniana }\end{array}$ & $\begin{array}{l}\text { Rosa } \\
\text { banksiae }\end{array}$ & $\begin{array}{l}\text { Rosa } \\
\text { indica }\end{array}$ & Mean \\
\hline M1= Sand $(100 \%)$ & 33.33 & 40.00 & 43.33 & 38.89 & $\begin{array}{c}43.91 \\
(41.45)\end{array}$ & $\begin{array}{c}85.92 \\
(68.16)\end{array}$ & $\begin{array}{c}96.29 \\
(83.51)\end{array}$ & $\begin{array}{c}75.37 \\
(64.37)\end{array}$ \\
\hline $\begin{array}{l}\text { M2=Sand+Manure } \\
(85 \%+15 \%)\end{array}$ & 40.00 & 43.33 & 56.67 & 46.67 & $\begin{array}{c}60.47 \\
(51.15)\end{array}$ & $\begin{array}{c}90.74 \\
(75.48)\end{array}$ & $\begin{array}{l}100.00 \\
(90.00)\end{array}$ & $\begin{array}{c}83.74 \\
(72.21)\end{array}$ \\
\hline $\begin{array}{l}\text { M3=Sand+Manure } \\
(70 \%+30 \%)\end{array}$ & 43.33 & 46.67 & 63.33 & 51.11 & $\begin{array}{l}63.05 \\
(52.58)\end{array}$ & $\begin{array}{c}93.33 \\
(77.71)\end{array}$ & $\begin{array}{l}100.00 \\
(90.00)\end{array}$ & $\begin{array}{c}85.46 \\
(73.43)\end{array}$ \\
\hline $\begin{array}{l}\text { M4=Sand+Manure+Soil } \\
(70 \%+15 \%+15 \%)\end{array}$ & 30.00 & 36.67 & 40.00 & 35.56 & $\begin{array}{c}47.62 \\
(43.63)\end{array}$ & $\begin{array}{l}89.63 \\
(74.65)\end{array}$ & $\begin{array}{l}96.29 \\
(83.51)\end{array}$ & $\begin{array}{l}77.85 \\
(67.26)\end{array}$ \\
\hline $\begin{array}{l}\text { M5 = Sand+Soil } \\
(85 \%+15 \%)\end{array}$ & 26.67 & 30.00 & 33.33 & 30.00 & $\begin{array}{l}40.65 \\
(39.60)\end{array}$ & $\begin{array}{c}85.92 \\
(68.16)\end{array}$ & $\begin{array}{l}96.29 \\
(83.51)\end{array}$ & $\begin{array}{c}74.29 \\
(63.76)\end{array}$ \\
\hline Mean & 34.67 & 39.33 & 47.33 & & $\begin{array}{c}51.14 \\
(45.68)\end{array}$ & $\begin{array}{c}89.12 \\
(72.83)\end{array}$ & $\begin{array}{l}97.78 \\
(86.10)\end{array}$ & \\
\hline C.D. $(p \leq 0.05)$ & $\begin{array}{l}\text { Growing medi } \\
\text { Genotype } \\
\text { Interaction }\end{array}$ & $\begin{array}{l}= \\
= \\
=\end{array}$ & $\begin{array}{l}9.7 \\
7.5\end{array}$ & & & & & $\begin{array}{c}7.762 \\
6.013 \\
\text { NS }\end{array}$ \\
\hline
\end{tabular}

During the present studies maximum survival of $82.38 \%$ was recorded with IBA $1000 \mathrm{ppm}$ and minimum survival of $66.35 \%$ was recorded under control. Significant interaction resulted in $100 \%$ survival of rooted cuttings of Rosa indica when treated with different concentrations of NAA (Table 3). The increase in survival percentage of rooted cuttings in the field might be due to more number of roots produced as a result of growth regulator treatment, which in turn absorbed more nutrients and moisture from the rooting medium and thus helped them in field establishment. Among the genotypes, 
maximum survival (96.98\%) was observed in Rosa indica and minimum survival $(43.38 \%)$ was observed in Rosa bourboniana. Higher survival of cuttings in Rosa indica is attributed to its better root number and length.

Propagation of rose rootstocks can be commercialized through the used of auxins. Present studies indicted that $1000 \mathrm{ppm}$ of IBA was optimum dose for improving rooting characteristics in Rosa bourboniana and Rosa banksiae. However, for Rosa indica, 1000 ppm of NAA gave better results.

Another experiment was laid out to evaluate the effect of different medium combination on the rooting characteristics of some genotypes of rose used as rootstocks for increasing their multiplication rate. A good growing medium would provide sufficient support for the plant and serve as a reservoir for nutrients and water. The physical and chemicals properties of medium influencing rooting and root growth of cuttings include porosity, water and air content, nutrient content, hormones and other metabolites. The nutritive capacity of the media (Abu-Hassan et al., 1994) and the concentration of hormones play an important part in rhizogenesis of cuttings (Ellyard and Ollerenshaw, 1984).

Influence of growing medium was significant on all rooting parameters. Minimum number of days taken to root initiation (22.13) was observed in cuttings when rooted in $\mathrm{M}_{3}$ medium (sand 70\%+manure 30\%) and minimum of 27.00 days in $\mathrm{M}_{5}$ medium (sand $85 \%+$ soil $15 \%$ ). This treatment was at par with $\mathrm{M}_{1}$ and $\mathrm{M}_{2}$. The decrease in time taken to root initiation might be due to the excellent drainage, good aeration and higher temperature in manure supplemented media. These results are in close conformity with the findings of Ochoa et al., (2003) in oleander cuttings and Dvin et al., (2011) in cuttings of MM111 apple. The minimum number of days taken to root initiation (22.70) was recorded in Rosa indica and maximum (25.02 days) in Rosa bourboniana (Table 4).

The highest rooting $(83.33 \%)$ of cuttings was observed in $\mathrm{M}_{3}$ medium (sand 70\%+manure $30 \%$ ) and lowest rooting $(54.44 \%)$ in $\mathrm{M}_{5}$ medium (sand $85 \%+$ soil $15 \%$ ). The increase in rooting percentage might be due to good aeration, high water holding capacity, excellent drainage and improved temperature in media containing sand and manure. Inclusion of soil into rooting media might have reduced the aeration and temperature of the medium which resulted in minimum rooting in soil supplemented media. The maximum rooting $(90.00 \%)$ was observed in Rosa indica and minimum rooting (53.33\%) in Rosa bourboniana. Rooting capacity of the cuttings vary due to genetic differences as some plants root profusely while others fail to root. Significant interaction resulted in $96.67 \%$ rooting in Rosa indica when rooted in $\mathrm{M}_{3}$ or $\mathrm{M}_{2}$ medium (Table 4).

Growing media significantly improved the root number and length of cuttings. Maximum number of primary roots (15.52) and length $\left(8.88 \mathrm{~cm}\right.$ ) was recorded in $\mathrm{M}_{3}$ medium (sand $70 \%+$ manure $30 \%$ ) followed by $\mathrm{M}_{2}$ medium (Table 5). Minimum primary root number (8.13) and root length $(5.34 \mathrm{~cm})$ was observed in $\mathrm{M}_{5}$ medium (sand $85 \%+$ soil $15 \%$ ). The increase in number of primary roots might be due to good drainage, aeration, better nutrition and water holding capacity of manure supplemented media. Rosa indica recorded the maximum number of primary roots (21.21) and root length $(10.10 \mathrm{~cm})$. Significant difference in root length among genotypes may be due to genetic characteristics of rootstocks or variation in auxin and carbohydrate content in cuttings.

Medium had a significant effect on the growth of new leaves on the cuttings (Table 6). 
Maximum number of cuttings with new leaves $(51.11 \%)$ was recorded in $\mathrm{M}_{3}$ medium (sand $70 \%+$ manure $30 \%$ ) followed by $\mathrm{M}_{2}$ medium (sand 85\%+ manure 15\%) and minimum (30.00\%) in $\mathrm{M}_{5}$ medium (sand 85\%+soil 15\%). Paramveer et al., (2011) also reported improved growth of anthurium in organic manure supplemented media compared to sand + soil medium. The increase in new leaf emergence might be due to high porosity and water holding capacity, good nutrient content and aeration of media containing sand and manure i.e. $\mathrm{M}_{2}$ and $\mathrm{M}_{3}$. Among the genotypes, new leaf emergence on cuttings was maximum $(47.33 \%)$ in Rosa indica and minimum $(34.67 \%)$ in Rosa bourboniana. Profuse rooting in Rosa indica produced better growth due to more uptake of nutrients and moisture from the growing medium and resulted in higher new leaf emergence. Field survival of rooted cuttings was significantly increased when cuttings were rooted in manure supplemented medium (Table 6). The maximum field survival of $85.46 \%$ and $83.74 \%$ was recorded in cuttings grown in manure supplemented media ie. $\mathrm{M}_{3}$ medium (sand $70 \%+$ manure $30 \%$ ) and $\mathrm{M}_{2}$ medium (sand $85 \%+$ manure $15 \%$ ). Field survival was minimum $(74.20 \%)$ in cuttings grown in $\mathrm{M}_{5}$ medium (sand 85\%+soil 15\%). The increase in field survival percentage might be due to the profuse root production of cuttings in manure rich media $\left(\mathrm{M}_{2}\right.$ and $\left.\mathrm{M}_{3}\right)$ having more nutrients, good aeration and water holding capacity. Field survival of cuttings was maximum (97.78\%) in Rosa indica followed by Rosa banksiae (89.12\%). Highest survival of Rosa indica might be attributed to its profuse fibrous root system.

Sand is usually recognized good medium for rooting of cuttings. However, supplementation of sand with some organic products has given better results (Bender and Nedim, 2007). In the present studies, rooting characteristics of cuttings were significantly improved when sand was used in combination with manure. Among the genotypes, Rosa indica gave significantly better results with respect to its rooting characteristics. It can be easily propagated at commercial scale through cuttings and used as rootstock for budding of commercial cultivars of rose.

\section{References}

Abu-Hassan, A.A., Zeawail, E.F. and Souidan, A.A. 1994. Effect of different planting substrates and dates on rooting potential of Ficus infectoria cuttings. Annals of Agricultural Sciences 32(2): 973-986.

Anderson, R.G. and Woods, T.A. 1999. An economic evaluation of single stem cut rose production. Acta Horticulturae 481: 629-34.

Arteca, R.N. 1996. Plant Growth Substances, Principles and Applications. Chapman and Hall, New York, pp: 127-145.

Bender, O.D. and Nedim, O. 2007. The effect of hazel nut husk compost and some organic and inorganic media on root growth of kiwifruit (Actinidia deliciosa). Journal of Agronomy 6: 113118.

Bose, T.K., Yadav, L., Pal, P., Das, P. and Parthasarathy, V.A. 2002. Commercial Flowers. Naya Prakash 2, Kailash Bose Street, Calcutta 1: 10-11.

Dvin, R.S., Moghadam, G.E. and Kiani, M. 2011. Rooting response of hardwood cuttings of MM111 apple clonal rootstock to indolebutyric acid and rooting media. Asian Journal of Applied Sciences 4(4): 453-458.

Ellyard, R.K and Ollerenshaw, P.J. 1984. Effect of indolebutryic acid, medium composition and cutting type on rooting of Grevillea johnsonii cutting at two basal temperatures. Combined Proceedings of the International Plant Propagators Society 34: 101-108. 
Gomez, K.A. and Gomez, A.A. 1983. Statistical Procedures for Agricultural Research. Johan Wiley and Sons, New York.

Hartmann, H.T., Kester, D.E., Davies, F.T. and Geneve, R.L. 2002. Plant Propagation: Principles and Practices, Prentice Hall India Pvt. Ltd., New Delhi.

Kaicker, U.S. and Dhayani, D. 1986. Performance of some indigenous and new exotic rose rootstocks in Delhi. Indian Rose Annual 5: 145-154.

Nanda, K.K. 1975. Physiology of adventitious root formation. Indian Journal of Plant Physiology 18: 80-87.

Ochoa, J., Banon, S., Fernandez, J.A., Franco, J.A. and Gonzalez, A. 2003. Influence of cutting position and rooting media on rhizogenesis of oleander cuttings. Acta Horticulturae 608: 101-106.

Pandey, S.N. and Sinha, B.K. 1997. Plant physiology. Vikas Publishing House Pvt. Ltd., New Delhi, India. pp 428-514. Paramveer, S., Dhaduk, B.K. and Chawla, S.L. 2011. Standardization of growing medium for anthurium cv. Flame under protected conditions. Indian Journal of
Horticulture 68(1): 86.90.

Patil, A.A. and Shirol, A.M. 1991. Studies on rooting of oleander cuttings. South Indian Horticulture 39: 48-53.

Rolston, S. H., Carlos, A. F. B. and Carlos, A. P. M. 1996. Adventitious root formation and development in cuttings of Mussaenda erythrophylla L. HortScience 31(6): 1023-1025

Singh, A.K., Singh, R., Ashutosh, M.K., Singh, Y.P. and Shiva, J. 2003. Effect of plant growth regulators on survival, rooting and growth characters in long pepper (Piper longum L.). Progressive Horticulture 1(1): 27-31.

Singh, B. and Dadlani, N.K. 1993. Rose breeding in India: Perspective and approaches. Journal of Ornamental Horticulture 1(1): 27-31.

Steel, G. D. and Torrie, J. H. 1981. Principles and Procedures of Statistics. McGraw Hill Book Company, Singapore.

Yashaswini, S., Venugopal, C.K. and Hegde, R.V. 2011. Propagation of a rare medicinal plant species Premna integrifolia by hardwood cuttings. Indian Journal of Horticulture 68(1):108-112

\section{How to cite this article:}

Sonam Dawa, Z. A. Rather, Tashi Stobgais, Tsering Angdus, Stanzin Lakdan and Phunstog Tundup. 2018. Effect of Growth Regulators and Growth Media on the Rhizogenesis of Some Genotypes of Rose through Stem Cuttings. Int.J.Curr.Microbiol.App.Sci. 7(01): 1138-1147. doi: https://doi.org/10.20546/ijcmas.2018.701.138 